\title{
ADAPTIVE BLOTCHES DETECTION FOR FILM RESTORATION
}

Antoni Buadès

\author{
MAP5, Univ. Paris Descartes \\ 45 rue des Saints Péres \\ 75270 Paris Cedex 06, France
}

Julie Delon, Yann Gousseau

\author{
LTCI, Télécom ParisTech \\ 46, rue Barrault \\ 75634 Paris Cedex 13, France
}

Simon Masnou

\author{
Institut Camille Jordan, Univ. Lyon 1 \\ 43, Boulevard du 11 novembre 1918 \\ 69622 Villeurbanne Cedex, France
}

\begin{abstract}
Blotches are very common, localized, and non persistent impairments in digitized film archive. Many methods have been proposed so far for detecting them and restoring the underlying regions. Most detection techniques rely on the hypothesis that blotches contradict a model of motion regularity and, up to a prior motion compensation, correspond to significant temporal variations of intensity with respect to a global threshold. In this paper, we propose a statistical approach to detect blotches in image sequences, which yields thresholds adapted to the local statistics of the frames, and which takes into account gray level differences in neighborhoods instead of isolated points. This approach is combined with a blockbased motion estimation. The whole procedure is confronted with classical approaches on several sequences.
\end{abstract}

Index Terms - Film restoration, Blotches, Adaptive detection, Statistical test, A contrario methods.

\section{INTRODUCTION}

Inevitable physical aging of film archive has dramatic consequences: the potential disappearing of a significant part of the world cultural heritage. Several programs have been funded in the past twenty years to transfer films and videos on a digital support in order to preserve and restore them. After digitization, there is a huge variety of impairments that may be seen on the resulting motion pictures. An exhaustive list can be found on the BRAVA project page ${ }^{1}$. We address in this paper the question of detecting and removing the so-called blotches in a digital sequence. Blotches are these non persistent, localized impairments usually due either to a loss of pieces of gelatin on the original film, or to the electrostatic adhesion of dust, hair, etc. that could not be cleaned out before digitization. There is a significant literature on this problem, that we will briefly survey in the next section. As we will see, the major problem is the definition of a criterion for detecting blotches because it can hardly be uniform on the whole

\footnotetext{
This work was supported by the French Agence Nationale de la Recherche (ANR), under the grant FREEDOM (ANR07-JCJC-0048-01), "Films, REstauration Et DOnnées Manquantes".

${ }^{1}$ http://brava.ina.fr/brava_public_impairments_list.en.html
}

image, but depends whether the pixels under examination lie on a quickly changing and highly contrasted region, or on a homogeneous region that does not change much in time. In order to avoid the so-called false alarms, i.e. the erroneous detection of uncorrupted pixels, many methods require the delicate tuning of several parameters.

In contrast, we propose in this paper an adaptive criterion for detecting blotches in image sequences. After a prior motion estimation by a block-based technique, we derive from the local distribution of intensity differences a local threshold above which a difference can hardly be due to noise, but more certainly to a blotch. The key point in this adaptive definition of blotchiness is inspired by a contrario approaches [2]. We illustrate at the end of the paper the efficiency and versatility of our approach on several examples.

\section{STATE OF THE ART}

A classical way to tackle the blotch removal problem is to proceed in three stages : motion estimation, blotch detection and restoration of the impaired regions. Each of these steps has been tackled in the literature with many different approaches. Surveys can be found in the book by A. Kokaram [6], R. Bornard's PhD thesis [1] and in the paper [4]. As for the motion estimation step, classical methods include variational approaches, block-based methods, prediction-correction methods and Bayesian methods. These latter usually perform well but at a rather high computational cost. In contrast, block-based methods, if implemented correctly, offers a reasonable tradeoff between efficiency and speed. Once the motion has been compensated, simple methods rely on a thresholding of various temporal coherence measures (SDIa [5], SDIp, ROD [7], sROD[9]). More involved approaches make use of Markov Random Fields to account for the spatial regularity of blotches [5]. Among thresholding methods, the sROD algorithm is known for its efficiency and we will recall its definition in the next section. There have been several attempts to tune properly the thresholds associated with these methods, using for instance an hysteresis [10], a prediction-correction technique [3], etc. We do not address in this paper the problem of blotch restoration, 
after the detection step, but here also many methods have been proposed in the literature: inpainting methods - either based on a PDE or on texture synthesis mode [1, 3], median filters (or more general rank filters), autoregressive models, parametric Markov random fields, etc. [1, 4]. Finally, observe that other approaches fuse the three aforementioned steps in a Bayesian framework, enabling a more robust estimation of motion and the handling of occlusions, see [4].

Before detailing the proposed detection methodology, we introduce some notations and recall two classical tests, SDIp and sROD.

\subsection{Notations}

We define now the notations and vocabulary that will be used throughout this paper: the film is denoted as $u=\left(u_{t}(x)\right)$, where $u_{t}$ is the frame at time $t$ and $x$ is the spatial variable on a fixed domain $\Omega$.

For each point $x$ of the current frame $u_{t}$, assume for the moment that we know $x^{-}$its corresponding point in the previous frame $u_{t-1}$ and $x^{+}$its corresponding point in the next frame $u_{t+1}$. Then we define $d^{-}(x)=u_{t}(x)-u_{t-1}\left(x^{-}\right)$and $d^{+}(x)=u_{t}(x)-u_{t+1}\left(x^{+}\right)$.

\subsection{Two classical tests}

Most of the classical tests consist in thresholding a function of $d^{+}$and $d^{-}$. The threshold is usually chosen globally in time and space. The oldest one, called SDIp [5], detects a blotch at $x$ if the quantity

$$
b(x)=\min \left(\left|d^{-}(x)\right|,\left|d^{+}(x)\right|\right) * \operatorname{sign}\left[d^{-}(x) * d^{+}(x)\right]
$$

is larger than a threshold $\alpha$. The so-called SDIa test does not impose a sign conservation, a simplification that significantly decreases the performances.

Other approaches rely on order statistics computed on the neighbors of $x^{+}$and $x^{-}$. The ROD detector [7] is known for its efficiency, but requires the delicate tuning of three threshold values. A simplified and very efficient version, called sROD, was introduced in [9]. Let us denote $\left(r_{1}, r_{2}, r_{3}\right)$ and $\left(r_{4}, r_{5}, r_{6}\right)$ the values of gray level observed in a column of three consecutive pixels centered respectively on $x^{+}$ and $x^{-}$. The point is detected as blotchy if one of the following conditions is met: $u(x)-\max \left(r_{1}, \ldots, r_{6}\right)>S$ or $\min \left(r_{1}, \ldots, r_{6}\right)-u(x)>S$, where $S$ is a positive constant.

\section{AN ADAPTIVE BLOTCH DETECTION}

In this section, we propose a simple statistical test to detect blotches in image sequences. Following the general ideas of a contrario approaches to image analysis [2], blotches are detected as image structures contradicting some simple null hypothesis. This test offers two advantages. First, it is adaptive, in the sense that it yields thresholds which adapt to the local statistics of the frames, and therefore vary spatially. Second, it is spatially coherent: gray level differences are observed in a whole neighborhood of the current point. Motion estimation will be discussed in section 4 and we assume for the moment that, for each pixel $x$ in the current frame $u_{t}$, the associated past and future points $x^{-}$and $x^{+}$in the previous and next frames are known. Before describing our test in detail, let us mention that an a contrario approach to blotch removal was previously used in [10], in order to disregard false alarms after a prior blotch detection using the sROD criterion. Our approach is completely different, since the statistical setting is used to drive the detection step itself.

\subsection{Null hypotheses}

For each pixel $x$, the goal is to decide whether the differences between the neighborhood of $x$ and the neighborhood of $x^{-}$ (or $x^{+}$) are meaningful, i.e. due to a blotch, or if they can be explained by the local statistics of the frames. The idea is to fix detection thresholds in such a way that patches following a naïve model $\mathcal{H}_{0}$ will not be detected as containing blotches.

Let $\mathcal{N}_{0}$ be a fixed neighborhood of 0 and $\left|\mathcal{N}_{0}\right|$ its size. For a point $x$ and a time $t$, consider the patch $u_{t}\left(x+\mathcal{N}_{0}\right)$ and its corresponding patch $u_{t-1}\left(x^{-}+\mathcal{N}_{0}\right)$ in the previous frame. The naïve model $\mathcal{H}_{0}^{x}$ (or null hypothesis) states that the point differences $u_{t}(x+y)-u_{t-1}\left(x^{-}+y\right)$, when y spans $\mathcal{N}_{0}$, are realizations of iid random variables following a centered normal distribution $\mathbb{N}\left(0, \sigma_{x}^{2}\right)$. We make the same assumption on the differences $u_{t}(x+y)-u_{t+1}\left(x^{+}+y\right)$, when $y$ spans $\mathcal{N}_{0}$.

\subsection{Decision criterion}

If the hypothesis $\mathcal{H}_{0}^{x}$ is satisfied, then the sum

$$
D_{x}^{-}:=\frac{1}{\left|\mathcal{N}_{0}\right|} \sum_{y \in \mathcal{N}_{0}}\left(u_{t}(x+y)-u_{t-1}\left(x^{-}+y\right)\right)
$$

is a realization of the distribution $\mathbb{N}\left(0, \sigma_{x}^{2} /\left|\mathcal{N}_{0}\right|\right)$, and the same result holds for $D_{x}^{+}$. The hypothesis $\mathcal{H}_{0}^{x}$ can thus be tested by computing $F_{x}\left(\left|D_{x}^{-}\right|\right)$and $F_{x}\left(\left|D_{x}^{+}\right|\right)$, where $F_{x}(\delta)$ denotes the tail of the Gaussian distribution

$$
F_{x}(\delta):=1-\frac{\sqrt{\left|\mathcal{N}_{0}\right|}}{\sqrt{2 \pi} \sigma_{x}} \int_{-\delta}^{\delta} e^{-\frac{\left|\mathcal{N}_{0}\right| s^{2}}{2 \sigma_{x}^{2}}} d s .
$$

If $F_{x}\left(\left|D_{x}^{-}\right|\right)$and $F_{x}\left(\left|D_{x}^{+}\right|\right)$are too small, i.e. if the observed differences $\left|D_{x}^{-}\right|$and $\left|D_{x}^{+}\right|$are too large, we can conclude that these differences can hardly be explained by the null hypothesis. In this case, $\mathcal{H}_{0}^{x}$ is rejected, which means that a blotch is detected at $x$.

Our adaptive spike detection index (ASDI) is defined as

$$
A S D I(x):=\max \left(F_{x}\left(\left|D_{x}^{-}\right|\right), F_{x}\left(\left|D_{x}^{+}\right|\right)\right) \cdot \operatorname{sign}\left(D_{x}^{-} \cdot D_{x}^{+}\right) .
$$

For a given threshold $\varepsilon$, the point $x$ in the frame $u_{t}$ is detected as a blotch if $0 \leq A S D I(x) \leq \frac{\varepsilon}{|\Omega|}$. Observe that the function 
$F_{x}$ only depends on the size of the patches $\mathcal{N}_{0}$ and on the local standard deviation $\sigma_{x}$. We will see in Section 4 how this standard deviation is estimated. The coefficient $\frac{1}{|\Omega|}$ is used to balance the number of tests per frame.

For a given threshold $\varepsilon$, this approach yields different adaptive thresholds on the absolute values $\left|D_{x}^{-}\right|,\left|D_{x}^{+}\right|$, when $x$ varies. Indeed, the smaller the value $\sigma_{x}$, the smaller the threshold on the absolute differences will be. In other words, a low contrasted blotch has a better chance to be detected on a flat background than on a highly textured background.

\section{TAKING MOTION INTO ACCOUNT}

\subsection{Block matching and blotch detection}

Our motion estimation scheme relies on block matching. In order to be fast, we divide the domain $\Omega$ into a fixed grid of overlapping blocks of size $(2 f+1)^{2}$. Neighboring blocks overlap over a band of width $f$ pixels. For each of these blocks $\mathcal{W}$ in the frame $u_{t}$, its best corresponding block in $u_{t-1}$ is denoted as $\mathcal{W}^{-}$. The best corresponding block is searched for over all blocks (not only on the fixed grid) in a region of size $(2 s+1)^{2}$. The best corresponding block is the one minimizing the $L^{2}$ distance between blocks. A best corresponding block $\mathcal{W}^{+}$in $u_{t+1}$ is computed in the same way.

The test ASDI is then applied to each pixel $x$ of $\mathcal{W}$, the standard deviation $\sigma_{x}$ being estimated empirically as the standard deviation of the pixel differences between $\mathcal{W}^{+}$and $\mathcal{W}^{-}$ (this standard deviation is thus the same for all points in $\mathcal{W}$ ). If the frame $u_{t}$ contains a blotch around $x$, this permits to avoid the blotch to affect the variance estimation. If a point $x$ belongs to several blocks, it is detected as a blotch if it is detected as such in at least one of these blocks.

\subsection{Speed-up}

In the motion estimation described above, the past and future blocks $\mathcal{W}^{-}$and $\mathcal{W}^{+}$associated with $\mathcal{W}$ are seeked in a restricted neighborhood of $\mathcal{W}$, i.e. a square of size $(2 s+1)^{2}$. The parameter $s$ should be larger than the largest motions between two successive frames, yielding heavy computations in presence of large motions. However, the whole process can be speeded up in the following way. The blotch detection is applied for increasing values of $s$, starting at $s=0$. At each step, the detection is restricted to the points detected at the previous value. This yields a major reduction of the computational cost. Another way to reduce the cost could be to remove from the sequence (before the detection step) the dominant motion due for instance to camera motion. This can be done with the approach of [8]. Observe also that common compression schemes incorporate block-wise motion estimations, that could be used for the detection procedure presented in this paper.

\section{EXPERIMENTS}

This section confronts the detector ASDI with SDIp and sROD on two sequences, called cmovie and super8. The experiments are obtained using the block-based implementation described in Section 4. Blocks are always chosen as squares of size $21 \times 21$, and the overlap between these blocks is chosen as half the size of a block (i.e. 10). The neighborhood $\mathcal{N}_{0}$ used for the test ASDI is a $3 \times 3$ centered square. As explained in Section 4.2, the detections are performed for increasing values of $s$ (maximum motion taken into account), starting from $s=0$ and up to $s=10$ for the sequence cmovie, and up to $s=30$ for the sequence super8.

Figure 1(a) shows one frame of the sequence cmovie, containing two low contrasted blotches, a bright spot and a dark string. As illustrated in Figures 1(c), 1(e), the dark string can be detected by SDIp and SROD using very low thresholds, but only at the price of many spurious detections. Besides, the dark blotch quickly disappears from detection maps when these thresholds are increased, while false detections persist (Fig. 1(d), 1(f)). On the contrary, ASDI correctly detects this blotch at $\varepsilon=1$, and $\varepsilon=10^{-2}$, while limiting the number of false detections, as illustrated in Figures $1(\mathrm{~g})$ and $1(\mathrm{~h})$. This experiment demonstrates the importance of the adaptive threshold, which yields better results here than the best thresholds of the classical tests.

Figure 2 illustrates the behavior of the three detectors on a frame of the sequence super8. ASDI detects all the blotches present in the frame at $\varepsilon=1$, in particular the two blotches on the left of the window and the scratch at the bottom of the frame, with a few false detections on the hands and arm of the man, due to motion. In comparison, SDIp and sROD give many false detections (especially on zones affected by motion) at thresholds for which they barely detect the first of the three aforementioned blotches.

\section{REFERENCES}

[1] R. Bornard. Probabilistic approaches for the digital restoration of television archives. $\mathrm{PhD}$ thesis, Ecole Centrale Paris, 2002.

[2] A. Desolneux, L. Moisan, and J.-M. Morel. Gestalt Theory and Image Analysis : A Probabilistic Approach. Lecture Notes in Mathematics. Springer, 2007.

[3] M. K. Güllü, O. Urhan, and S. Ertürk. Blotch detection and removal for archive video restoration. In ISCIS, pages 739$747,2005$.

[4] A. C. Kokaram. On missing data treatment for degraded video and film archives: a survey and a new bayesian approach. IEEE Transactions on Image Processing, 13(3):397-415, 2004.

[5] A.C. Kokaram, R.D. Morris, W.J. Fitzgerald, and P.J.W. Rayner. Detection of missing data in image sequences. Image Processing, IEEE Transactions on, 4(11):1496-1508, Nov 1995. 


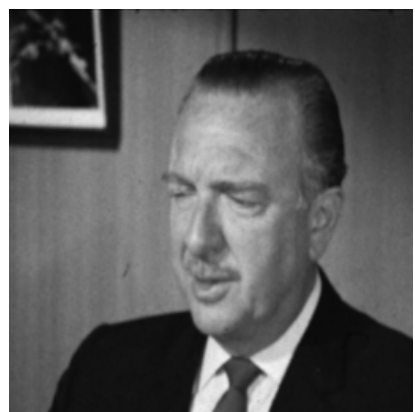

(a) One frame of cmovie

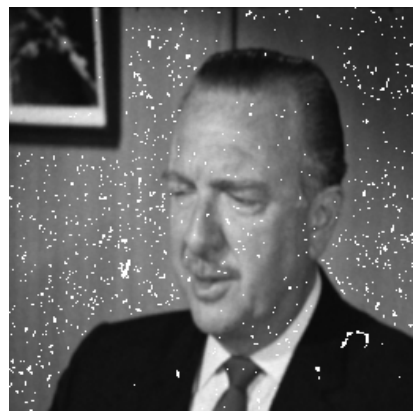

(e) $\mathrm{sROD}, S=1$

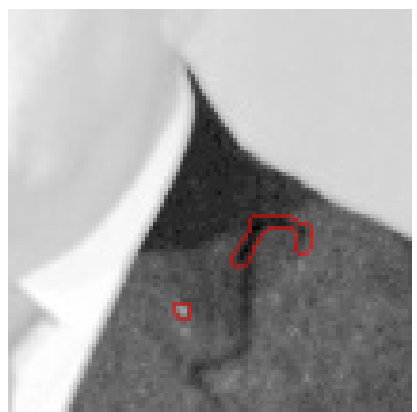

(b) Extract of Fig. 1(a), with a contrast change, revealing two blotches.

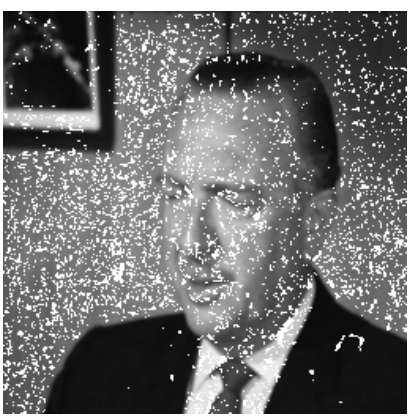

(c) SDIp, $\alpha=2$

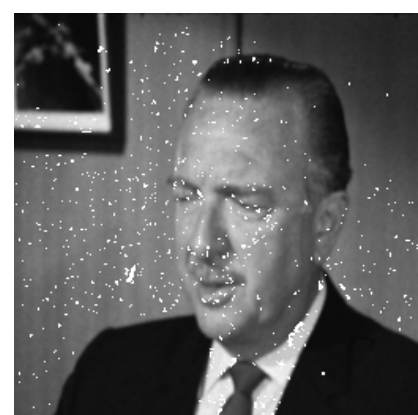

(d) SDIp, $\alpha=5$

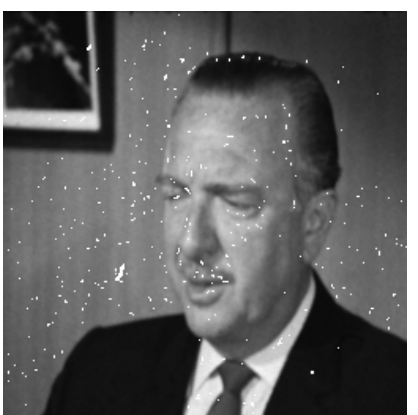

(f) sROD, $S=4$

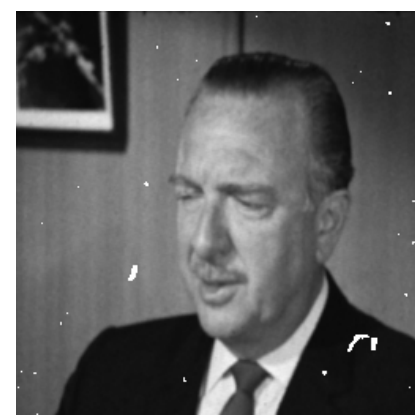

(g) ASDI, $\varepsilon=1$

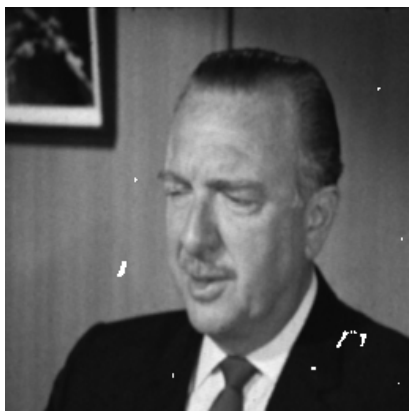

(h) ASDI, $\varepsilon=10^{-2}$

Fig. 1. Adaptive blotch detection: Figure 1(b) shows a subpart of Figure 1(a) with a change of contrast, revealing two low contrasted blotches, a bright spot and a dark string (surrounded by red lines). The other figures show the results of SDIp, sROD and ASDI (proposed in this paper) for different thresholds. For SDIp and SROD, the low contrasted blotches quickly disappear as the threshold increases, while many false detections remain. In contrast, ASDI is much more robust.

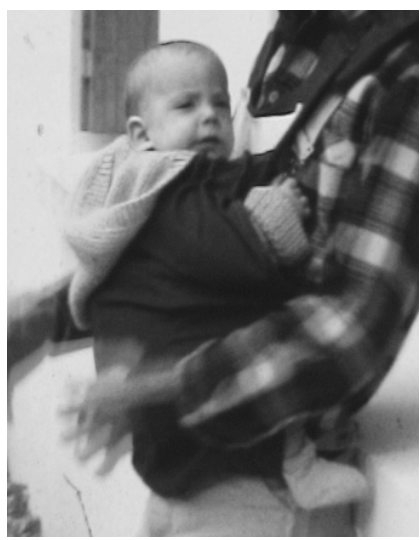

(a) One frame of super 8

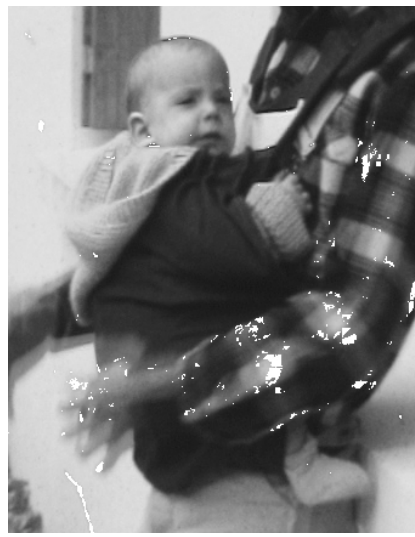

(b) SDIp, $\alpha=15$

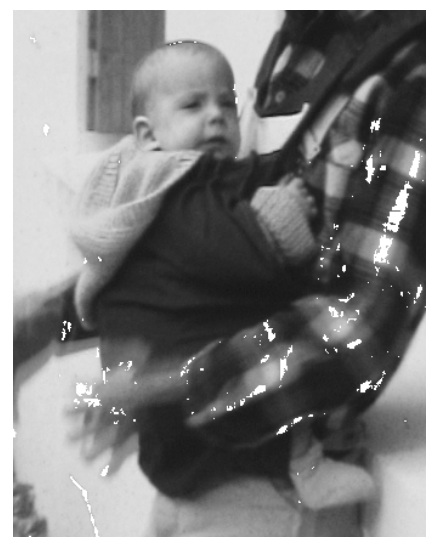

(c) $\mathrm{sROD}, S=15$

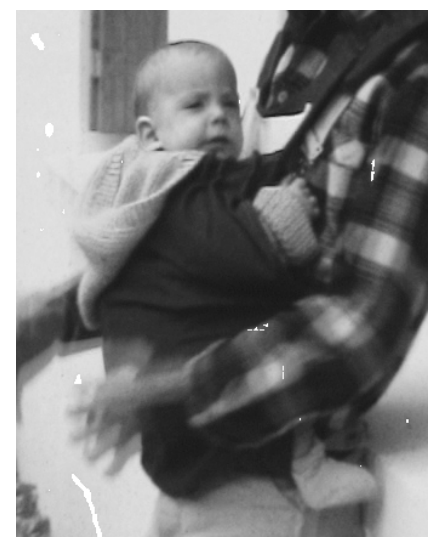

(d) ASDI, $\varepsilon=1$.

Fig. 2. Another example where SDIp and sROD miss some blotches and return many false detections whereas ASDI gives satisfactory results (original blotches and their detection can be better seen when zooming in on a digital version of the paper).

[6] Anil C. Kokaram. Motion Picture Restoration: Digital Algorithms for Artefact Suppression in Degraded Motion Picture Film and Video. Springer-Verlag, London, UK, 1998.

[7] M. J. Nadenau and S. K. Mitra. Blotch and scratch detection in image sequences based on rank ordered differences. In Proc. 5th Int. Workshop on Time-Varying Image Processing and Moving Object Recognition, 1996.

[8] J. M. Odobez and P. Bouthemy. Robust multiresolution esti- mation of parametric motion models. Journal of Visual Communication and Image Representation, 6(4):348 - 365, 1995.

[9] P. M. B. Van Roosmalen. Restoration of archived film and video. PhD thesis, Delft University of Technology, 1999.

[10] S. Tilie, L. Laborelli, and I. Bloch. A contrario false alarms removal for improving blotch detection in digitized films restoration. In EC-SIPMCS, IWSSIP, pages 739-747, june 2007. 